\title{
Aktueller Stand der Therapieforschung bei pathologischem Kaufen
}

\author{
A. Müller M. de Zwaan \\ Abteilung für Psychosomatik und Psychotherapie, Universitätsklinikum Erlangen, Deutschland
}

Schlüsselwörter

Pathologisches Kaufen · Kaufsucht · Zwanghaftes Kaufen Impulskontrollstörung

\begin{abstract}
Zusammenfassung
Unter pathologischem Kaufen wird die fehlangepasste starke Beschäftigung mit Kaufen bzw. ein als unwiderstehlich und/oder sinnlos wahrgenommener Kaufdrang oder Kaufexzess verstanden. Es handelt sich um ein Phänomen, welches erhebliche psychische Belastungen und deutliche soziale und finanzielle Probleme verursacht und welches nicht ausschließlich in Phasen einer Manie oder Hypomanie auftritt. Obwohl zirka 6-8\% der Erwachsenen in Deutschland als stark kaufsuchtgefährdet bezeichnet werden können, wird Kaufsucht im klinischen Alltag vermutlich häufig übersehen. Der bisherige Kenntnisstand basiert auf einer vergleichsweise geringen Anzahl wissenschaftlicher Studien. Die eindeutige klassifikatorische Einteilung ist bisher noch nicht gelungen. Am ehesten kann das pathologische Kaufen als Impulskontrollstörung verstanden und als «nicht näher bezeichnete abnorme Gewohnheit und Störung der Impulskontrolle" (ICD-10 F 63.9) diagnostiziert werden. Klinische Studien zeigen eine hohe Komorbidität zu affektiven Störungen, Angststörungen, Substanzabhängigkeiten, Essstörungen, Zwangsstörungen und anderen Störungen der Impulskontrolle, vor allem zum pathologischen Glücksspiel. Ätiologie und Pathogenese des pathologischen Kaufens sind noch unklar, wobei von einer Reihe psychologischer, sozialer und biologischer Faktoren ausgegangen wird. Die Erfolg versprechenden Ergebnisse offener Studien mit Antidepressiva (vor allem selektiven Serotonin-Wiederaufnahmehemmern, SSRI) oder Opiatantagonisten konnten bisher durch zwei randomisierte kontrollierte Studien nicht bestätigt werden. Das erste störungsspezifische, kognitiv-behaviorale Gruppentherapiekonzept wurde 2000 in den USA publiziert. Eine kontrollierte Studie zur Wirksamkeitsüberprüfung dieser Therapie wird zur Zeit durchgeführt. In Anlehnung an dieses Therapiemanual wurde 2003 in der Abteilung für Psychosomatik und Psychotherapie des Universitätsklinikums Erlangen mit einer kontrollierten Psychotherapiestudie begonnen.
\end{abstract}

\section{Key Words}

Pathological buying · Addictive buying - Compulsive buying · Impulse control disorder

\section{Summary}

Current Status of Psychotherapy Research on Pathological Buying

Pathological or 'compulsive' buying is defined by the presence of maladaptive preoccupations with buying, impulses to buy which are experienced as irresistible or senseless, and by excessive buying behavior. The buying preoccupations, impulses, or behaviors cause marked distress and result in social and financial problems. The behavior does not occur exclusively during periods of hypomania or mania. Even though pathological buying has been estimated to affect $6-8 \%$ of the general adult population in Germany, it is frequently overlooked in clinical routine. The literature on this topic is still small, although, in the past decade, there has been renewed interest in disorders characterized by behavioral excess. There is still an ongoing debate about the appropriate classification of pathological buying; however, it seems to be best linked to impulse control disorders and should be diagnosed as 'impulse control disorder not otherwise specified' (ICD 10 F 63.9). Research has shown it to be associated with significant psychiatric comorbidity particularly mood and anxiety disorders, substance use disorders, eating disorders, obsessive-compulsive disorders, and disorders of impulse control, particularly pathological gambling. The etiology and pathogenesis of pathological buying is unknown, but there is evidence that psychological, social, and biological factors are involved. There is no standard approach to the treatment of pathological buying. The positive results of open trials with antidepressants (usually selective serotonine reuptake inhibitors, SSRIs) and opioid antagonists so far could not be confirmed in 2 randomized controlled trials. A disorder-specific cognitive-behavioral group treatment manual was published in the United States, in 2000 , a controlled study is currently being conducted. A similar trial using this manual was started in 2003 at the Department of Psychosomatics and Psychotherapy at the University Hospital in Erlangen.

\begin{tabular}{ll}
\hline KARGER & ๑ 2004 S. Karger GmbH, Freiburg \\
Fax +49 7614520714 & Accessible online at: \\
$\begin{array}{l}\text { E-mail Information@Karger.de } \\
\text { www.karger.com }\end{array}$ & www.karger.com/ver
\end{tabular}




\section{Einleitung}

Die «krankhafte Kauflust» oder «Oniomanie» wurde bereits Anfang des Jahrhunderts von Kraepelin [1909] und Bleuler [1923] beschrieben. Wesentlich später interessierten sich kanadische, amerikanische und deutsche Konsumforscher für dieses Phänomen und seine Nachweisbarkeit mittels quantitativer Erhebungsinstrumente [Valence et al., 1988; Faber und O’Guinn, 1988, 1989; Scherhorn et al., 1990]. Etwa zeitgleich erschienen Kasuistiken psychodynamischer Therapien Betroffener [Winestine, 1985; Krueger, 1988; Lawrence, 1990]. Seitdem wurden mehrere wissenschaftliche Arbeiten zur Phänomenologie, Ätiologie und Komorbidität publiziert [McElroy et al., 1994; Christenson et al., 1994; Schlosser et al., 1994; Black, 1996]. Die eindeutige klassifikatorische Einteilung des pathologischen Kaufens ist bisher noch nicht gelungen, was sich in den verschiedenen Bezeichnungen widerspiegelt. Während im amerikanischen Sprachraum der Begriff «compulsive buying» (zwanghaftes Kaufen) bevorzugt wird, kursiert in den deutschen Medien eher die Bezeichnung «Kaufsucht». Mitunter wird das Phänomen auch als «impulsives Kaufen» bezeichnet. Die Autorinnen bevorzugen den umfassenderen Begriff «pathologisches Kaufen» [Hand, 1998].

Im Folgenden wird das Phänomen näher beschrieben und der derzeitige Kenntnisstand sowie Therapieansätze werden skizziert.

\section{Phänomenologie}

\section{Frau $X$}

Frau X ist 52 Jahre alt, ledig und lebt mit ihrer 13-jährigen Tochter zusammen. Sie arbeitet Vollzeit als Sekretärin.

Sie liebe «schöne, farbenfrohe, ausgefallene, auffallende Anziehsachen». Sie werde eigentlich nicht von dem Bedürfnis, unbedingt etwas erwerben zu müssen, getrieben. Meistens wolle sie einfach ein bisschen die Wohnung verlassen, und wenn sie dann durch die Stadt bummele und «was Schönes» sehe, dann gehe ihr «Herz auf» und sie müsse es mitnehmen. Dabei handele es sich nicht um teure Ware, alles sei sehr preisgünstig oder sogar «billig». Fast täglich kaufe sie ein, hinzu kämen unzählige Katalogbestellungen. Die angehäufte Ware quelle aus den Schränken und Kisten. Ihre Wohnung gleiche einem «besseren Basar». Mittlerweile habe sie zirka 80 Sommer- und 90 Winterjacken, 200 Hosen und rund 500 Paar Schuhe. Das könne sie niemals alles anziehen. Wenn es ihr «ganz furchtbar» gehe, verspüre sie kein Kaufinteresse. Aber wenn sie sich «so miesepetrig» fühle, dann sei sie gefährdet. Angefangen habe alles vor 25 Jahren mit ihrem ersten Gehalt. Gedanken an die immensen Schulden, welche sich inzwischen auf mindestens 50000 Euro belaufen, verdränge sie.

\section{Frau $Y$}

Frau $\mathrm{Y}$ ist 55 Jahre alt, seit 25 Jahren verheiratet und hat vier erwachsene Kinder. Sie arbeitet halbtags als Arzthelferin.

Trotz Überschuldung (35000 Euro) habe sie immer weiter eingekauft. Wegen unbezahlter Rechnungen sei eine Bewährungsstrafe gegen sie verhängt worden. Sie habe keinen Zugriff mehr auf die Familienkonten. Bis dahin habe sie ungefähr zwei Mal pro Woche exzessiv eingekauft. Sie habe nur «exklusive, teure Sachen», vorzugsweise Kleidungsstücke, für sich, den Ehemann und vieles für die Kinder und Enkel erworben. Beim Einkaufen habe sie in «einer anderen Welt» gelebt und im Übrigen die
Gespräche mit den Verkäuferinnen in den edlen Geschäften sehr genossen. Die meisten Waren benutze sie überhaupt nicht. Sehr viele Kleidungsstücke lägen noch in der Originalverpackung mit Preisschildern in den Schränken, unterm Bett oder auf dem Dachboden. Nun könne sie zwar nichts mehr kaufen, aber sie gehe weiterhin in die Stadt und lasse sich in exklusiven Geschäften beraten und die Ware zurückhängen, die sie dann telefonisch absage.

\section{Frau $Z$}

Frau $\mathrm{Z}$ ist 34 Jahre alt, ledig und hat eine 16-jährige Tochter. Sie arbeitet Vollzeit als Berufsbetreuerin.

Sie werde den ganzen Tag von Gedanken ans Kaufen geplagt, die sie einfach nicht loswerden könne. Dauernd schwirrten ihr Gedanken an «praktische Dinge» wie Wäschetrockner, Regale, Mikrowellen und Ähnliches durch den Kopf. Dann gehe sie auf Schnäppchenjagd, vergleiche Angebote, suche nach dem günstigsten Angebot, fahre sämtliche Läden ab, verfalle in «Jagdfieber». Manchmal relativiere sich der Kaufwunsch während des Suchens. Meistens werde sie jedoch fündig bei ihrer «Trophäensammlung». Besonders günstige Waren erwerbe sie gleich mehrfach, um sie dann eventuell weiterzuverschenken. Wenn sie mal kein «Ziel» im Kopf habe, müsse sie in Katalogen stöbern, bis sie eines gefunden habe. $\mathrm{Zu}$ Spontankäufen käme es selten. Die Frequenz der Kaufattacken habe über die Jahre nicht zugenommen, allerdings seien die Artikel teurer geworden, mit jeder Gehaltserhöhung etwas preisintensiver. Die Ware häufe sich bei ihr zu Hause an. Die Zimmer, der Keller, die Garage seien mit Kartons «vollgemüllt». Wenn sie mal nicht zum Einkaufen käme, fühle sie sich gereizt, angespannt, «umhergetrieben». Mittlerweile habe sie 50000 Euro Schulden. Sie habe bemerkt, dass Kaufhandlungen häufig durch «Freude» ausgelöst würden. Wenn sie sich freue oder euphorisch sei, dann könne sie nicht widerstehen.

Pathologisches Kaufen kann sich unterschiedlich manifestieren. Manche Betroffene erwerben vor allem preisgünstige oder vergünstigte Ware, andere bevorzugen exklusive Markenartikel. Mitunter kommt es zu multiplen Einkäufen der gleichen Ware. Es werden phasenhafte Kaufattacken berichtet oder auch tägliche Einkäufe. Während manche Betroffene das Kaufen per Katalog, TV oder Internet vorziehen, genießen andere den direkten Kontakt zum Verkaufspersonal. Immer werden unnötige Dinge oder eine nicht adäquate Anzahl von Dingen konsumiert, die hinterher kaum oder gar nicht benutzt werden und die sich dann fast immer anhäufen. Oft werden die Kaufgüter weitergegeben oder der Erwerb wird verheimlicht. Häufig wird der Kauf von Geschenken für andere beschrieben. Dabei scheinen Frauen Kleidung, Schuhe, Schmuck, Kosmetik oder Küchenartikel zu bevorzugen. Männer favorisieren eher Technikartikel, Autozubehör, Sportartikel oder Antiquitäten. Meistens erwachsen wegen der exzessiven, unangemessenen Einkäufe zum Teil immense Schulden. Mitunter werden sogar Strafverfahren anhängig.

\section{Theoretischer Hintergrund}

\section{Definition und Diagnostik}

Susan McElroy et al. [1994] formulierten für «compulsive buying» folgende diagnostische Kriterien, welche auf einer Untersuchung von 20 Betroffenen basieren und analog $\mathrm{zu}$ den 
DSM-IV-Kriterien für Impulskontrollstörungen definiert wurden [McElroy et al., 1992, 1994]:

A Fehlangepasste starke Beschäftigung mit Erwerben bzw. Kaufen oder fehlangepasste Erwerbs- bzw. Kaufimpulse bzw. Kaufverhaltensweisen, auf die mindestens eine der folgenden Beschreibungen zutrifft:

a. Häufige, starke Beschäftigung mit Kaufen oder Kaufimpulsen, welche als unwiderstehlich, sich aufdrängend, intrusiv und/oder sinnlos wahrgenommen wird.

b. Häufiges Kaufen von mehr, als man sich leisten kann. Häufiges Kaufen von Dingen, die nicht benötigt werden. Kaufen über längere Zeitperioden als geplant.

B Der Kaufdrang, die Kaufimpulse oder Kaufverhaltensweisen verursachen erhebliches Leiden. Sie sind zeitaufwendig, beeinträchtigen deutlich die sozialen und beruflichen Funktionen oder haben finanzielle Probleme zur Folge (Verschuldung oder Konkurs).

C Die exzessiven Erwerbs- oder Kaufverhaltensweisen treten nicht ausschließlich in Phasen einer Manie oder Hypomanie auf.

Ein wichtiges Abgrenzungskriterium zum «normalen» Kaufen stellen die negativen Folgen des pathologischen Kaufens dar [O’Guinn und Faber, 1989; Faber, 1992; Lejoyeux et al., 1996; Black, 2001]. Betroffene berichten häufig von zum Teil immenser Verschuldung, manchmal strafrechtlichen Konsequenzen und oft familiärer Zerrüttung. Insofern rechtfertigen die erheblichen negativen sozialen Folgen die Beurteilung des pathologischen Kaufens als eine Störung mit Krankheitswert.

Es existieren mittlerweile mehrere Erhebungsinstrumente zur quantitativen Beurteilung des pathologischen Kaufens. In Deutschland erlaubt der Hohenheimer Kaufsuchtindikator von Scherhorn et al. [1990] eine reliable und valide Erfassung der Kaufsuchtgefährdung. Der Fragebogen besteht aus 16 Items. Die Veröffentlichung des Handbuchs wird derzeit vorbereitet. Der Hohenheimer Kaufsuchtindikator stellt eine modifizierte und für Deutschland adaptierte Variante der zuvor in Kanada entwickelten Compulsive Buying Original Measurement Scale dar [Valence et al., 1988]. In amerikanischen Studien werden vorzugsweise die aus 7 Items bestehende Compulsive Buying Scale (CBS) von Faber und O'Guinn [1992] oder die Compulsive Acquisition Scale (CAS) von Frost et al. [1998] verwendet, welche 18 Items abfragt. Zur Einschätzung des Schweregrads und zur Verlaufsmessung gibt es noch keine validierten Instrumente. In einigen Untersuchungen kam die Yale-Brown Obsessive Compulsive Scale - Shopping Version (Y-BOCS-SV) von Monahan und Black [1996] zum Einsatz. Die Y-BOCS-SV ist eine weiterentwickelte Version der bekannten Y-BOCS zur Erfassung von Denk- und Handlungszwängen. Es bleibt jedoch fraglich, ob mit einem Instrument, das zur Einschätzung von Zwangsstörungen konzipiert wurde, auch das pathologische Kaufen adäquat abgebildet werden kann. Zur Verlaufserfassung dient daher meist die durch Selbstbeobachtung und/oder Interview erfasste Frequenz einzelner Episoden pathologischen Kaufens [Müller et al., 2004].

\section{Klassifikation}

Bereits Kraepelin und Bleuler haben das pathologische Kaufen zu den Impulskontrollstörungen gezählt. Auch nach den diagnostischen Kriterien von McElroy et al. gehört das Phänomen zu den Störungen der Impulskontrolle. Andere verstehen pathologisches Kaufen als stoffungebundene Sucht [in Deutschland z.B. Grüsser et al., 2004; Scherhorn et al., 1990; Gross, 2003]. Das Konzept der so genannten «Verhaltenssüchte» muss jedoch kritisch diskutiert werden. Es wird von einer Ausdünnung des definitorischen Gehalts von Sucht gesprochen.

Plausibel erscheint die von Hollander und Wong [1995] vorgeschlagene Einordnung des pathologischen Kaufens in das Modell der Zwangsspektrumsstörungen. Dabei wird das pathologische Kaufen ebenfalls den Impulskontrollstörungen zugerechnet. In diese Gruppe gehören außerdem das pathologische Spielen, die Trichotillomanie, die sexuellen Zwänge, das selbstverletzende Verhalten und die Kleptomanie. Bei dem Versuch, die Zwangsspektrumsstörungen dimensional auf einem Kontinuum zwischen Zwangsstörungen (risk avoidance) und Impulskontrollstörungen (risk seeking) aufzureihen, würde das pathologische Kaufen eher zu den Impulskontrollstörungen tendieren.

Wie bereits eingangs erwähnt, ist die eindeutige klassifikatorische Einteilung des pathologischen Kaufens bisher nicht gelungen. Der derzeitige Forschungsstand erlaubt nicht, das pathologische Kaufen als eigenständiges Störungsbild zu betrachten. Hier bedarf es weiterer wissenschaftlicher Studien. In Anlehnung an die bisherigen wissenschaftlichen Arbeiten schlagen die Autorinnen vor, pathologisches Kaufen nach ICD-10 als «nicht näher bezeichnete abnorme Gewohnheit und Störung der Impulskontrolle» (ICD-10 F 63.9) zu diagnostizieren.

\section{Epidemiologie}

Wie andere Zwangsspektrumsstörungen auch bleibt pathologisches Kaufen als «heimliches Leiden» zumeist relativ lange Zeit unbemerkt. Aufmerksamkeit erregen erst die oft erheblichen negativen Folgen. Bisher existieren nur wenige bevölkerungsrepräsentative Befragungen. Die - bezogen auf die Datenbasis weltweit umfangreichste Studie stammt von einer interdisziplinären Forschungsgruppe der Universität Stuttgart-Hohenheim [Scherhorn et al., 1990, 1994]. Im Rahmen der Kaufsuchtforschung wurden 1991 über 1500 Deutsche in den alten und neuen Bundesländern mit dem Hohenheimer Kaufsuchtindikator befragt. Exakt 10 Jahre später wurde die Befragung mit über 1000 Deutschen wiederholt [Neuner und Reisch, 2002; Reisch et al., 2004]. Die erste Untersuchung ergab, dass zirka $5 \%$ der erwachsenen Bevölkerung in den alten und zirka 1\% der Bevölkerung in den neuen Bundesländern als «stark kaufsuchtgefährdet» eingestuft werden konnten. Die zweite, aktuellere Befragung zeigte eine erhebliche Zunahme und weit gehende Angleichung der Kaufsuchtgefährdung in Ost- und 
Tab. 1. Ergebnisse zur Lebenszeitprävalenz (\%) komorbider Störungen bei pathologischem Kaufen, erhoben mit dem SCID

\begin{tabular}{|c|c|c|c|c|c|c|c|c|}
\hline & Stichprobe: $\mathrm{n}$ & $\begin{array}{l}\text { Affektive } \\
\text { Störung }\end{array}$ & MD & $\begin{array}{l}\text { Angst- } \\
\text { störung }\end{array}$ & $\begin{array}{l}\text { Substanz- } \\
\text { missbrauch }\end{array}$ & $\begin{array}{l}\text { Zwangs- } \\
\text { störung }\end{array}$ & Essstörung & BED \\
\hline \multirow[t]{2}{*}{ Christenson et al., 1994} & CB: 24 & 54 & 50 & $50 *$ & $46^{*}$ & 13 & $21 *$ & 17 \\
\hline & NB: 24 & 50 & 46 & 21 & 13 & 0 & 4 & 0 \\
\hline \multirow[t]{2}{*}{ Black et al., 1998} & CB: 33 & $64 * *$ & $61 *$ & 42 & 21 & 3 & 15 & n.a. \\
\hline & NB: 22 & 27 & 27 & 27 & 9 & 0 & 5 & \\
\hline
\end{tabular}

SCID = Structured Clinical Interview for DSM-III-R; CB = compulsive buyer; BED = binge eating disorder; MD = major depression;

$\mathrm{NB}=$ normal buyer; n.a. $=$ nicht angegeben.

$* \mathrm{p}<0,05 ; * \mathrm{p} \leq 0,01$.

Westdeutschland. In den neuen Bundesländern hatte sich die Zahl der Kaufsuchtgefährdeten nahezu versechsfacht. Mittlerweile müssen zirka $8 \%$ der Erwachsenen in den alten und zirka $6 \%$ in den neuen Bundesländern als «stark kaufsuchtgefährdet» bezeichnet werden [Reisch et al., 2004]. Eine frühere amerikanische Untersuchung mit der CBS hatte eine Häufigkeit um $8 \%$ ergeben, wobei eine wesentlich kleinere repräsentative Stichprobe $(\mathrm{n}=292)$ befragt worden war [Faber und O'Guinn, 1992]. Diese einmaligen Befragungsergebnisse mit Screeninginstrumenten können natürlich nicht mit einer klinischen Diagnose des pathologischen Kaufens gleichgesetzt werden. Zwar weisen insbesondere die Ergebnisse der Hohenheimer Studie auf die sehr hohe Tendenz zu unkontrollierten, pathologischen Käufen in der deutschen Bevölkerung hin. Bei genauerer Betrachtung bleibt die Prävalenz des pathologischen Kaufens jedoch unbekannt. Hier sind weitere wissenschaftliche Arbeiten erforderlich, um das Ausmaß pathologischen Kaufens genau zu erfassen.

Die deutschen Studien zeigen, dass prinzipiell alle Bevölkerungs- und Einkommensschichten betroffen zu sein scheinen. Die höchsten Werte für Kaufsuchtgefährdung wurden 2001 bei Personen mit mittlerer bis guter Bildung sowie bei Personen mit niedrigem Einkommen gefunden, was interpretationsbedürftig bleibt [Neuner und Reisch, 2002; Reisch et al., 2004]. Außerdem scheinen nach diesen Ergebnissen jüngere Menschen gefährdeter als ältere zu sein und Frauen scheinen eher betroffen als Männer. Sowohl die amerikanischen als auch die deutschen repräsentativen Studien unterstützen die These, dass pathologisches Kaufen ein tendenziell weibliches Phänomen ist [Reisch und Neuner, 2002]. In klinischen Studien wurde ein Frauenanteil zwischen 80 und $90 \%$ gefunden [McElroy et al., 1994; Christenson et al., 1994; Schlosser et al., 1994; Lejoyeux et al., 1999].

\section{Komorbidität}

In der Literatur wird eine hohe Komorbidität zwischen pathologischem Kaufen und Depressionen, Angststörungen, Substanzabhängigkeiten, Essstörungen, Störungen der Impulskontrolle und Zwangsstörungen berichtet [Schlosser et al.,
1994; McElroy et al., 1994; Black et al., 2000; Mitchell et al., 2002; Ninan et al., 2000]. Allerdings sind nur zwei kontrollierte Studien bekannt [Christenson et al., 1994; Black et al., 1998] (Tab. 1). Christenson et al. [1994] verglichen 24 pathologische mit 24 «normalen» Käufer/innen mittels Strukturiertem Klinischen Interview für DSM-III-Diagnosen (SCID) [Spitzer et al., 1989]. Bei pathologischen Käufer/innen konnten signifikant häufiger Substanzmissbrauch, Angststörungen und Essstörungen (Lebenszeitprävalenz) sowie andere Impulskontrollstörungen diagnostiziert werden. Black et al. [1998] untersuchten 33 pathologische und 22 «normale» Käufer/innen mit dem SCID und fanden bei den pathologischen Käufer/innen signifikant häufiger affektive Störungen.

Am Universitätsklinikum Erlangen wurden im Rahmen einer kontrollierten Psychotherapiestudie bisher 22 pathologische Käuferinnen mit dem SCID für DSM-IV-Diagnosen [Wittchen et al., 1997] untersucht. Es wurden ebenfalls hohe Lebenszeitprävalenzen für Angststörungen, vor allem soziale Phobie (64\%), Depressionen (55\%) und auch für Zwangsstörungen (41\%) gefunden [Müller et al., 2004]. Die Ergebnisse wurden jedoch noch nicht mit einer Kontrollgruppe verglichen.

In der Literatur finden sich auch Hinweise auf eine hohe Komorbidität mit Persönlichkeitsstörungen. Schlosser et al. [1994] berichteten mit 22\% Lebenszeitprävalenz ein relativ hohes Vorkommen einer zwanghaften Persönlichkeitsstörung bei 46 mit dem SCID für Persönlichkeitsstörungen [Pfohl et al., 1987] untersuchten pathologischen Käufer/innen. Bei 15\% diagnostizierten sie eine Borderline-Persönlichkeitsstörung, und $15 \%$ der Patienten/innen erfüllten die Kriterien einer vermeidenden Persönlichkeitsstörung.

Außerdem scheint es einen engen Zusammenhang zwischen pathologischem Kaufen und zwanghaftem Horten zu geben [Frost et al., 1998; Coles et al., 2003; Steketee und Frost, 2003]. Beides gehört nach Frost et al. [1998] zum Konstrukt «zwanghafter Erwerb» (compulsive acquisition). Horten scheint nahezu immer mit vermehrten Käufen einherzugehen. Pathologisches Kaufen ist aber nicht notwendigerweise mit Horten verbunden. Wie häufig Horten unter pathologischen Käufer/ innen tatsächlich auftritt, ist noch nicht systematisch untersucht worden. 
In einigen klinischen Studien wurde pathologisches Kaufen als komorbide Störung eines anderen primär untersuchten Beschwerdebildes erfasst. Lejoyeux et al. [1995, 1997, 1999] fanden pathologische Kaufverhaltensweisen bei Depressiven. Faber et al. [1995] erhoben mit der CBS bei Patientinnen mit einer Binge Eating Disorder (BED) $(\mathrm{n}=84)$ signifikant häufiger pathologisches Kaufen als bei Patientinnen ohne BED (n = 113). Du Toit et al. [2001] untersuchten 85 Patienten/innen mit einer Zwangsstörung mit einem strukturierten klinischen Interview zur Erfassung zwanghaft-impulsiver Spektrumsstörungen (SCID-OCSD) und diagnostizierten bei 10,6\% der Patienten/innen pathologisches Kaufen.

Pathologisches Kaufen wird ebenso als komorbide Störung bei pathologischen Spieler/innen berichtet. Specker et al. [1995] interviewten 40 pathologische Spieler/innen und 64 Kontrollpersonen. Die pathologischen Spieler/innen litten signifikant häufiger unter pathologischem Kaufen mit einer Lebenszeitprävalenz von $25 \%$.

\section{Pathogenese}

Es wird angenommen, dass an der Entstehung von pathologischem Kaufen eine Reihe psychologischer, sozialer und biologischer Faktoren beteiligt sind. Bisher liegen keine Forschungsergebnisse über spezifische Faktoren vor.

Wie bei anderen Zwangsspektrumsstörungen auch wird eine pathogenetische Rolle des serotonergen Systems vermutet. Allerdings liegen noch keine Befunde vor, die diese These eindeutig stützen würden [Devor et al., 1999].

Soziale Beeinflussung geschieht durch Geschlechtsrollen, Normen, Werte, Sozialisationsbedingungen, Kompensationsmöglichkeiten und anderes [Faber, 1992; Reisch et al., 2004]. Die meisten Autoren gehen von einer psychischen Disposition für pathologisches Kaufen aus. Scherhorn et al. [1994] vermuteten, dass ein niedriges Selbstwertgefühl und eine ausgeprägte Fantasieorientierung die Vulnerabilität erhöhen. O'Guinn und Faber [1989] berichteten höhere Materialismuswerte [nach Belk, 1985] bei pathologischen Käufer/innen.

Neben psychischen Dispositionen bzw. Persönlichkeitsfaktoren (traits) müssen auch situationale Bedingungen (states) und die Funktionalität der Verhaltensexzesse berücksichtigt werden. Defizite in der Selbstregulation können in Anlehnung an die sozial-kognitive Lerntheorie von Bandura [1991] als ursächlich für pathologisches Kaufverhalten angenommen werden [Baumeister und Vohs, 2004; Faber, 2004].

In der Literatur wird zumeist der kompensatorische Charakter der Käufe hervorgehoben [Lejoyeux et al., 1999]. Miltenberger et al. [2003] schlussfolgerten aus einer Selbstbeobachtungsstudie mit 17 pathologischen Käufer/innen, dass negative Gefühle pathologisches Kaufen auslösen. Während des Kaufaktes hätten die Käufer/innen verstärkt euphorische Emotionen protokolliert. Anschließend sei es zu einer Wiederverschlechterung der aversiven Gefühle gekommen. Die euphori- schen Gefühle während des Kaufens und die anschließende Verschlechterung können als aufrechterhaltende Determinanten verstanden werden.

Pathologisches Kaufen kann also als ein inadäquater Bewältigungsversuch für aversive Stimmungen angesehen werden. In der Folge bemerken Betroffene kurzfristig eine Verbesserung ihrer Stimmung. Längerfristig stabilisiert sich jedoch die negative Stimmung, und Schuldgefühle, Depressionen sowie das Gefühl des Kontrollverlusts belasten die Käufer/innen. Einige Autoren nehmen an, dass es Subtypen pathologischer Käufer/innen gibt, nämlich zwanghafte und impulsive [Nataraajan und Goff, 1991; Faber, 2004]. Zwanghaftes pathologisches Kaufen werde durch negative Gefühle und Distress ausgelöst, während impulsives pathologisches Kaufen eher durch euphorische Stimmungen veranlasst werde.

\section{Therapie}

\section{Medikamentöse Therapie}

Die Erfolg versprechenden Ergebnisse der wenigen offenen Medikamentenstudien bzw. der wenigen berichteten Kasuistiken konnten bisher durch kontrollierte Studien nicht bestätigt werden [Bullock und Koran, 2003]. Es werden erfolgreiche Therapien mit Antidepressiva (vor allem serotoninen Wiederaufnahmehemmern, SSRI), zum Teil auch in Kombination mit Phasenprophylaktika, beschrieben [McElroy et al., 1991, 1994; Lejoyeux et al., 1995; Black et al., 1997; Koran et al., 2002]. Andere Autoren berichten von einer erfolgreichen Behandlung mit dem Opiatantagonisten Naltrexon [Kim, 1998; Grant, 2003].

Zwei kontrollierte Studien von Ninan et al. [2000] und Black et al. [2000] mit bis zu $300 \mathrm{mg}$ Fluvoxamin pro Tag ergaben keinen Unterschied zwischen Medikament und Placebo. Sowohl die Patienten/innen der Versuchs- als auch der Placebogruppe hatten sich hinsichtlich der Kaufsymptomatik deutlich verbessert. Beide Studien berichteten im Übrigen von sehr hohen Abbruchraten von nahezu 50\%.

Koran et al. [2003] behandelten 24 pathologische Käufer/ innen 7 Wochen lang mit Citalopram maximal $60 \mathrm{mg}$ pro Tag. 15 Patientinnen zeigten eine deutliche Verringerung der Kaufattacken. Diese Responder wurden anschließend in einem doppelblinden placebokontrollierten Versuchsplan weitere 9 Wochen lang untersucht. Die Patientinnen, die weiterhin Citalopram erhielten, zeigten keine Rückfälle. Dagegen waren 5 der 8 placebobehandelten Frauen rückfällig. Daraus schlussfolgern Koran et al., dass Citalopram ein effektives Medikament zur Behandlung pathologischen Kaufens sei.

\section{Psychotherapie}

In der Literatur finden sich einige Kasuistiken psychodynamischer [Winestine, 1985; Krueger, 1988; Lawrence, 1990] und 
verhaltenstherapeutischer [Bernik et al., 1996; Bongers, 2000] Behandlungen Betroffener. Allerdings wurden bisher keine systematischen kontrollierten Psychotherapiestudien publiziert. Das erste störungsspezifische, kognitiv-behaviorale Therapiekonzept zur Behandlung pathologischer Käufer/innen stammt von Burgard und Mitchell aus den USA [2000]. Erste Ergebnisse einer offenen Studie sprechen für den Erfolg dieses Therapieangebots. Eine kontrollierte Untersuchung wird derzeit in den USA durchgeführt, wobei noch keine Resultate veröffentlicht wurden. Am Universitätsklinikum Erlangen wurde im Herbst 2003 in der Abteilung für Psychosomatik und Psychotherapie mit einer kontrollierten Psychotherapiestudie begonnen. Insgesamt sollen 60 pathologische Käufer/innen einbezogen werden. Die Betroffenen werden randomisiert Versuchs- und Warte-Kontrollgruppen zugeordnet. Um die Wirksamkeit der Therapie überprüfen zu können, erfolgt eine eingehende psychologische Diagnostik zu Behandlungsbeginn und -ende sowie bei einem Follow-up 6 Monate nach Behandlungsende. Tabelle 2 gibt eine Übersicht über die verwendeten Erhebungsinstrumente. Die ambulante, kognitiv-behaviorale Gruppentherapie orientiert sich an dem störungsspezifischen Therapiekonzept von Burgard und Mitchell. Tabelle 3 skizziert anhand einer Stundenübersicht die Themen der einzelnen Sitzungen. Es handelt sich um eine Kurzzeitintervention mit insgesamt 12 wöchentlich stattfindenden Gruppensitzungen zu jeweils 90 Minuten. Den Patientinnen wird in adäquater Form der derzeitige Kenntnisstand über pathologisches Kaufen vermittelt. Es werden individuelle (Kauf-)Verhaltensanalysen erstellt, Geldmanagement erlernt und alternative «gesunde» Kaufverhaltensweisen entwickelt. In der Therapie wird schwerpunktmäßig mit folgenden verhaltenstherapeutischen Techniken gearbeitet: Selbstbeobachtung, Verhaltensanalysen, Erlernen von Stimuluskontrolle und Selbstkontrolltechniken, kognitive Umstrukturierung und graduierte Exposition mit Reaktionsmanagement.

Die innerhalb der Erlanger Studie erfolgte klinische Arbeit mit Betroffenen zeigt, dass besonderes Augenmerk auf die konsequente Durchführung von Selbstbeobachtungstechniken (Kaufprotokolle) und Verhaltensanalysen gelegt werden muss. Nach Erfahrung der Autorinnen ist die Tendenz zur Verleugnung oder Rationalisierung der pathologischen Kaufverhaltensweisen bei den Patientinnen extrem hoch. Zwar verfügen die Betroffenen allein auf Grund der externen negativen Konsequenzen (Schulden, Strafanzeigen) des pathologischen Kaufens über eine hohe Änderungsmotivation, gleichwohl torpedieren die häufig sehr ausgeprägten kognitiven Vermeidungsstrategien die gewünschten Verhaltensänderungen. Vor diesem Hintergrund erscheint das geschlossene, störungsspezifische Gruppensetting sehr hilfreich, weil die «Kaufexpertinnen» sich gegenseitig mit den zum Teil sehr subtilen Vermeidungstechniken und maladaptiven Verhaltensweisen konfrontieren können.

Neben den therapeutischen Ansätzen können Betroffene sicher auch von Selbsthilferatgebern profitieren. Im deutschen
Tab. 2. Erhebungsinstrumente der Erlanger Psychotherapiestudie zum pathologischen Kaufen

\begin{tabular}{ll}
\hline Erhebunsinstrument & Autor/innen \\
\hline Hohenheimer Kaufsuchtindikator, GABS & Scherhorn et al., 1990 \\
Compulsive Buying Scale, CBS & Faber und O’Guinn, 1992 \\
Compulsive Acquisition Scale, CAS & Frost et al., 1998 \\
Yale-Brown Obsessive Compulsive & \\
Scale-Shopping Version, Y-BOCS-SV & Monahan und Black, 1996 \\
Saving Inventory-Revised, SI-R & Coles und Frost, 2003 \\
Maudsley Zwangsinventar, MOC & Hodgson u. Rachman, \\
& 1994 \\
Barratt Impulsiveness Scale, BIS-11 & Barratt, 2000 \\
Neo-Fünf-Faktoren Inventar, NEO-FFI & Borkenau und Ostendorf, \\
& 1993 \\
Symptom-Checkliste, SCL-90-R & Franke, 1995
\end{tabular}

Strukturiertes Klinisches Interview für DSM-IV für Achse I und II,

SKID I und II

Wittchen et al., 1997

Tab. 3. Behandlungsprogramm beim pathologischen Kaufen

\begin{tabular}{cl}
\hline Stunde & Inhalt \\
\hline & Einführung \\
1 & Überblick über die Behandlung \\
& Was ist pathologisches Kaufen? \\
2 & Erkennen pathologischer Kaufverhaltensweisen \\
& Erwartete Verhaltensänderungen \\
& Pro und Contra pathologisches Kaufen \\
3 & Auslöser und Konsequenzen \\
& Änderungsstrategien \\
4 & Geldmanagement \\
& «Gesundes» Kaufen \\
5 & Horten \\
6 & Reaktionen: Gedanken, Gefühle, Verhalten \\
7 & Kognitive Umstrukturierung \\
8 & Auslöser und Gedankenketten \\
9 & Selbstwert \\
10 & Exposition und Reaktionsmanagement \\
& Stressmanagement \\
11 & Problemlösen \\
12 & Rückfallprävention \\
& Resümee und Ausblick
\end{tabular}

Sprachraum steht die Übersetzung des amerikanischen Selbsthilferatgebers von Mohr-Catalano und Soneberg [1996] zur Verfügung, dessen Wirksamkeit jedoch noch nicht empirisch überprüft wurde.

\section{Schlussfolgerung}

Beim pathologischen Kaufen handelt es sich um ein vermutlich verbreitetes Phänomen, das bei den Betroffenen und 
ihrer Umgebung hohen Leidensdruck verursacht. Wahrscheinlich wird es im klinischen Alltag häufig übersehen. Der bisherige Kenntnisstand basiert auf einer vergleichsweise geringen Anzahl wissenschaftlicher Studien. Weitere Untersuchungen sind notwendig, um ein besseres Verständnis und wirkungsvolle Therapieansätze zu entwickeln. Am Universitätsklinikum Erlangen wurde im Herbst 2003 mit einer kontrollierten Psychotherapiestudie begonnen. Mit ersten Ergebnissen kann im Laufe des kommenden Jahres gerechnet werden.

\section{Literatur}

Bandura A: Cognitive theory of self-regulation. Organ Behav Hum Dec 1991;50:248-287.

Barrat E: Barrat Impulsiveness Scale, Version 11 (BIS11); in American Psychiatric Association (ed): Handbook of Psychiatric Measures. Washington, DC, APA, 2000, pp 691-693.

Baumeister RF, Vohs KD: Handbook of Self-Regulation. Research, Theory, and Applications. New York, Guildford, 2004.

Belk R: Materialism: Trait aspects of living in the material world. J Consum Res 1985;12:265-280.

Bernik MA, Akerman D, Amaral JAMS, Braun RCND: Cue exposure in compulsive buying. J Clin Psychiatry 1996;57:90.

Black DW: Compulsive buying: A review. J Clin Psychiat 1996;57:50-55.

Black DW: Compulsive buying disorder. Definition, assessment, epidemiology and clinical management. CNS Drugs 2001;15:17-27.

Black DW, Monahan P, Gabel J: Fluvoxamine in the treatment of compulsive buying. J Clin Psychiatry 1997:58:159-163.

Black DW, Repertinger S, Gaffney GR, Gabel J: Family history and psychiatric comorbidity in persons with compulsive buying: Preliminary findings. Am J Psychiatry 1998;155:960-963.

Black DW, Gabel J, Hansen J, Schlosser S: A doublebind comparison of fluvoxamine versus placebo in the treatment of compulsive buying disorder. Ann Clin Psychiatry 2000;12:205-211.

Bleuler E: Lehrbuch der Psychiatrie, ed 4. Berlin, Springer, 1923.

Bongers A: Fallbeschreibung einer verhaltenstherapeutisch orientierten Behandlung von Kaufsucht; in Poppelreuter S (Hrsg): Nicht nur Drogen machen süchtig: Entstehung und Behandlung von stoffungebundenen Süchten. Weinheim, Beltz, 2000, pp 165180

Borkenau P, Ostendorf F: Neo-Fünf-Faktoren-Inventar (NEO-FFI) nach Costa und McCrae. Handanweisung. Göttingen, Hogrefe, 1993.

Bullock K, Koran L: Psychopharmacology of compulsive buying. Drugs Today 2003;39:695-700.

Burgard M, Mitchell JE: Group cognitive-behavioral therapy for buying disorder; in Benson A: I Shop, therefore I Am: Compulsive Buying and the Search for Self. New York, Jason Aronson, 2000, pp 367-397.

Christenson GA, Faber RJ, de Zwaan M, Raymond NC, Specker SM, Ekern MD, Mackenzie TB, Crosby RD, Crow SJ, Eckert ED, Mussell MP, Mitchell JA: Compulsive buying: Descriptive characteristics and psychiatric comorbidity. J Clin Psychiatry 1994;55: 5-11.

Coles ME, Frost RO, Heimberg RG, Steketee G: Hoarding behaviors in a large college sample. Behav Res Ther 2003;41:179-194.

Devor EJ, Magee HJ, Dill-Devor RM, Gabel J, Black DW: Serotonin transporter gene (5-HTT) polymorphisms and compulsive buying. Am J Med Genet 1999; 88:123-125.
Du Toit PL, v Kradenburg J, Niehaus D, Stein DJ: Comparison of obsessive-compulsive disorder patients with and without comorbid putative obsessive-compulsive spectrum disorders using a structured clinical interview. Compr Psychiatry 2001;42:291-300. Faber RJ: Money changes everything. Compulsive buying from a biopsychosocial perspective. Am Behav Sci 1992;35:809-819.

Faber RJ: Self-control and compulsive buying; in Kasser T, Kanner A (eds): Psychology and Consumer Culture: The Struggle for a Good Life in a Materialistic World. Washington, DC, APA, 2004, pp 169-187. Faber RJ, O'Guinn TC: Dysfunctional consumer socialization: A search for the roots of compulsive buying. 13th Annual International Association for Research in Economic Psychology Colloquium. Leuven (Belgium), 1988.

Faber RJ, O'Guinn TC: Classifying compulsive consumers: Advances in the development of a diagnostic tool. Adv Consum Res 1989;16:738-744.

Faber RJ, O'Guinn TC: A clinical screener for compulsive buying. J Consum Res 1992;19:459-469.

Faber RJ, Christenson GA, deZwaan M, Mitchell J: Two forms of compulsive consumption: Comorbidity of compulsive buying and binge eating. J Consum Res 1995;22:296-304

Franke GH: SCL-90-R. Die Symptom-Checkliste von Derogatis. Deutsche Version. Manual. Göttingen, Beltz, 1995.

Frost RO, Kim HJ, Morris C, Bloss C, Murray-Close M, Steketee G: Hoarding, compulsive buying and reasons for saving. Behav Res Ther 1998;36:657-664.

Grant JE: Three cases of compulsive buying treated with naltrexone. Int J Psychiatry Clin Pract 2003;7:223225

Gross W: Sucht ohne Drogen. Frankfurt/M., Fischer, 2003.

Grüsser SM, Thalemann C, Albrecht U: Exzessives, zwanghaftes Kaufen oder «Verhaltenssucht»? Ein Fallbeispiel. Wien Klin Wochenschr 2004;116:201-204.

Hand I: Pathologisches Kaufen - Kaufzwang, Kaufrausch oder Kaufsucht?; in Lenz G, Demal U, Bach M (Hrsg): Spektrum der Zwangsstörungen. Wien, Springer, 1998, pp 123-132.

Hodgson RJ, Rachman S: Maudsley Obsessional Compulsive Inventory; in Fischer J, Corcoran K (eds): Measures for Clinical Practice: A Sourcebook. New York, Free Press, 1994, pp 340-341.

Hollander E, Wong CM: Introduction: Obsessive-compulsive spectrum disorders. J Clin Psychiatry 1995;56: 3-6.

Kim SW: Opioid antagonists in the treatment of impulse-control disorders. J Clin Psychiatry 1998;59:159_ 164.

Koran LM, Bullock KD, Hartston HJ, Elliott MA, D'Andrea V: Citalopram treatment of compulsive shopping: An open-label study. J Clin Psychiatry 2002; 63:704-708.

Koran LM, Chuong HW, Bullock KD, Smith SC: Citalopram for compulsive shopping disorder: An open-label study followed by double-blind discontinuation. J Clin Psychiatry 2003;64:793-798.
Kraepelin E: Psychiatrie. Ein Lehrbuch für Studierende und Ärzte, I. Band: Allg. Psychiatrie. Leipzig, Johann Ambrosius Barth, 1909.

Krueger DW: On compulsive shopping and spending: A psychodynamic inquiry. Am J Psychother 1988;42: 574-584.

Lawrence L: The psychodynamics of the compulsive female shopper. Am J Psychoanal 1990;50:67-70

Lejoyeux M, Hourtané M, Adès J: Compulsive buying and depression. J Clin Psychiatry 1995;56:38.

Lejoyeux M, Adès J, Tassain V, Solomon J: Phenomenology and psychopathology of uncontrolled buying. Am J Psychiatry 1996;153:1524-1529.

Lejoyeux M, Tassain V, Solomon J, Adès J: Study of compulsive buying in depressed patients. J Clin Psychiatry 1997;58:169-173

Lejoyeux M, Haberman N, Solomon J, Ades J: Comparison of buying behavior in depressed patients presenting with or without compulsive buying. Compr Psychiatry 1999;40:51-56.

McElroy SL, Satlin A, Pope HG, Keck PE, Hudson JJ: Treatment of compulsive shopping with antidepressants. A report of three cases. Ann Clin Psychiatry 1991;3:199-204.

McElroy SL, Hudson JI, Pope HG, Keck PE, Aizley HG: The DSM-III-R impulse control disorders not elsewhere classified: Clinical characteristics and relationship to other psychiatric disorders. Am J Psychiatry 1992;149:318-327.

McElroy SL, Keck PE, Pope HG, Smith JMR, Strakowski SM: Compulsive buying: A report of 20 cases. J Clin Psychiatry 1994;55:242-248.

Miltenberger RG, Redlin J, Crosby R, Stickney M, Mitchell J, Wonderlich S, Faber R, Smyth J: Direct and retrospective assessment of factors contributing to compulsive buying. J Behav Ther Exp Psychiatry 2003; 34:1-9.

Mitchell JA, Redlin J, Wonderlich S, Crosby R, Faber R, Milotenberger R, Smyth J, Stickney M, Gosnell B, Burgard M, Lancaster K: The relationship between compulsive buying and eating disorder. Int J Eat Disord 2002;32:107-111.

Mohr-Catalano E, Sonenberg N: Kaufen, kaufen, kaufen ... Wegweiser für Menschen mit zwanghaftem Kaufverhalten. Ein Selbsthilfeprogramm mit vielen Fragebögen und Protokollen. Regeln fürs Geldmanagement. Stuttgart, Thieme, 1996

Monahan P, Black DW, Gabel J: Reliability and validity of a scale to measure change in persons with compulsive buying. Psychiatry Res 1996;64:59-67.

Müller A, Silbermann A, Kadegge-Schindler, de Zwaan M: Pathologisches Kaufen: Deskriptive Charakteristika, sozio-demographische Daten und psychiatrische Komorbidität bei 22 Patientinnen. Psychother Psychosom Med Psychol 2004;54:104.

Nataraajan R, Goff BG: Compulsive buying: Toward a reconceptualization. J Soc Behav Pers 1991;6:307-326. Neuner M, Reisch L: Zur Entwicklung der Kaufsucht in Deutschland: Bericht über eine laufende Studie; in: Die Sparkasse. Berlin, Deutscher Sparkassen- und Giroverband, 2002;119:40-43. 
Ninan PTN, McElroy S, Kane CP, Knight BT, Casuto LS, Rose SE, Marsteller FA, Nemeroff CB: Placebocontrolled study of fluvoxamine in the treatment of patients with compulsive buying. J Clin Psychopharmacol 2000;20:362-366.

O'Guinn TC, Faber RJ: Compulsive buying: A phenomenological exploration. J Consum Res 1989;16: 147-157.

Pfohl B, Blum N, Zimmermann M, Stangl D: Structured Interview for DSM-III Personality Disorders, Revised. Jowa City, JA, University of Iowa, 1987.

Reisch L, Neuner M, Raab G: Zur Entstehung und Verbreitung der «Kaufsucht» im vereinten Deutschland. Aus Politik und Zeitgeschichte 2004:16-22.

Reisch L, Neuner M: Woman and addictive buying: The gender question revisited; in Ureta IG, Fernandez EO (eds): El consumo y la adicción a las compras: Diferentes perspectivas. Bilbao, The University of the Basque Country Press, 2002, pp 169-195.

Scherhorn G, Reisch LA, Raab G: Addictive buying in West Germany: An empirical study. J Consum Policy. 1990;13:355-387.

Scherhorn G, Reisch LA, Raab G: Kaufsucht. Bericht über eine empirische Untersuchung; in Arbeitspapier 50. Universität Hohenheim, 1994.

Schlosser S, Black DW, Repertinger S, Freet D: Compulsive buying. Demography, phenomenology, and comorbidity in 46 subjects. Gen Hosp Psychiatry 1994;16: 205-212.

Specker SM, Carlson GA, Christenson GA, Marcotte M: Impulse control disorders and attention deficit disorder in pathological gamblers. Ann Clin Psychiatry 1995;7:175-179.

Spitzer RL, Williams JBW, Gibbon M, First MB: Structured Clinical Interview for DSM-III-R, Revised (SCID). New York, Biometric Research New York State Psychiatric Institute, 1989.

Steketee G, Frost R: Compulsive hoarding: Current status of the research. Clin Psychol Rev 2003;23: 905927.

Valence G, d'Astous A, Fortier L: Compulsive buying: Concept and measurement. J Consum Policy 1988;11: 419-433.

Winestine MC: Compulsive shopping as a derivate of a childhood seduction. Psychoanal Q 1985;54:70-72.

Wittchen HU, Zaudig M, Fydrich T: Strukturiertes Klinisches Interview für DSM-IV. Achse I und II. Göttingen, Hogrefe, 1997. 